\title{
The effect of intravitreal injections on dry eye, and proposed management strategies
}

REVIEW

This article was published in the following Dove Press journal:

Clinical Ophthalmology

16 August 2017

Number of times this article has been viewed

\author{
Augustinus Laude ${ }^{1-3}$ \\ Jimmy WK Lim ${ }^{1,2}$ \\ Vishwanath Srinagesh ${ }^{4}$ \\ Louis Tong ${ }^{2,5-7}$ \\ 'National Healthcare Group Eye \\ Institute, Tan Tock Seng Hospital, \\ ${ }^{2}$ Singapore Eye Research Institute, \\ ${ }^{3}$ Lee Kong Chian School of Medicine, \\ Nanyang Technological University, \\ Singapore; ${ }^{4}$ Krieger Eye Institute, \\ Baltimore, MD, USA; ${ }^{5}$ Singapore \\ National Eye Centre, ${ }^{6}$ Duke NUS \\ Medical School, ${ }^{7}$ Yong Loo Lin School \\ of Medicine, National University of \\ Singapore, Singapore
}

\begin{abstract}
Intravitreal injection of anti-vascular endothelial growth factor (anti-VEGF) agents has become a commonly used treatment method for a number of ophthalmic conditions, including age-related macular degeneration. Although anti-VEGF therapy has shown promising results for many patients, there are several aspects of its application that have not been thoroughly investigated. One of these is the development and/or escalation of concurrent dry eye syndrome. Many patients undergoing treatment are already predisposed to dry eye disease due to their age and overall ocular health. As dry eye can have a substantial impact on quality of life, it has become increasingly apparent that the clinical signs and symptoms should be closely monitored and aggressively managed. This will allow for the optimization of patient comfort and visual potential. Here, we discuss the reasons why dry eye may develop during the course of repeated ocular anti-VEGF therapy, highlighting the key concerns about current practices and proposing possible solutions to improve the outcome for the patients.
\end{abstract}

Keywords: age-related macular degeneration, povidone-iodine, toxicity, ocular health, chronic ophthalmic treatment

\section{Introduction}

Dry eye syndrome is a chronic inflammatory ocular surface disease. Typical symptoms include burning and itchiness, gritty sensation, tearing, redness of the conjunctiva, foreign body sensation, and blurred vision. In addition to the ocular surface discomfort, it is often accompanied by intermittent blurred vision and other potential ocular surface damages. Worsening of these symptoms may occur during reading, using a computer, driving at night, and carrying out professional work. ${ }^{1}$

Dry eye syndrome has been associated with several clinical markers including tear hyperosmolarity, elevated inflammatory markers, and abnormal tear production. ${ }^{2}$ The onset of hyperosmolarity can be driven by a number of different factors and diseases, such as age, hormonal imbalances, and diabetes. It is known to cause damage to the surface epithelium of the eye by activating a cascade of inflammatory events at the ocular surface releasing inflammatory mediators such as interleukin-1 $\alpha$ (IL-1 $\alpha$ ), tumor necrosis factor $\alpha$ (TNF- $\alpha$ ), vascular endothelial growth factor (VEGF), and other cytokines into the tears. These inflammatory agents in turn lead to epithelial cell death by apoptosis, resulting in loss of the goblet cells that propagates to a reduction of mucus secretion that under normal circumstances protects the eye against both shear stress and chemical damage. ${ }^{3}$

Dry eye syndrome is also a commonly experienced side effect in patients receiving repeated intravitreal injections that may be often overlooked. Below we discuss in more detail the reasons for the development of dry eye syndrome, how intravitreal 
injection therapy affects the health of the treated eye, and what could be the future avenues to relieve the discomfort associated with dry eye syndrome.

\section{Discussion}

\section{Mechanism of dry eye and AMD}

Dry eye is a common complaint in patients who are undergoing chronic ophthalmic treatments or have a predisposition to altered or damaged corneal nerves (eg, in the form of increased tortuosity or reduced nerve fiber length). This may be observed in older patients, patients with long-standing diabetes, or patients who have received extensive retinal surgery. Nevertheless, it appears that age and age-related diseases are the most prominent factors playing into the development of the symptoms of dry eye. Age-related macular degeneration (AMD) is a common ocular condition and a leading cause of vision loss and blindness among people aged 50 years and older. ${ }^{4}$

AMD causes damage to the macula that is responsible for sharp central vision. As AMD progresses, blurred or blank spots in central vision may develop. AMD may be categorized as atrophic or neovascular. While atrophic AMD leads to a gradual loss of vision, neovascular AMD progresses faster and is the most advanced form of the disease responsible for $90 \%$ of all AMD-related blindness. For a long time, neovascular AMD was considered untreatable and two-thirds of those affected were expected to be legally blind within 2 years of developing the disease. ${ }^{5}$

Neovascular AMD (also called the exudative AMD) involves choroidal neovascularization and associated retinal edema, which is largely driven by the overproduction of VEGF. ${ }^{6}$ Patients suffering from neovascular AMD produce high levels of VEGF, which promote growth of abnormal blood vessels underneath the retina that leak fluid which ultimately decreases central vision. To combat these effects, a common practice today for treating neovascular AMD patients is to perform anti-VEGF injection therapy, which typically requires multiple monthly injections into the affected eye. The use of anti-VEGF drugs has nearly halved the incidence of AMD-related blindness in some countries. ${ }^{5}$

\section{Anti-VEGF agents and their mechanism of action}

Anti-VEGF agents have been increasingly used in the management of AMD in recent years. In general, anti-VEGF agents used in therapy are either monoclonal antibodies (bevacizumab), ${ }^{7}$ antibody derivatives/fragments against VEGF (ranimizumab), ${ }^{8}$ or VEGF trap (aflibercept). The mechanism of anti-VEGF agents lies in their ability to decrease the levels of active VEGF in the eye by binding to the molecule and blocking/neutralizing its effects, thus reducing abnormal angiogenesis and leakage, which concomitantly stabilize vision loss and, in some cases, may also improve sight. Studies show that repeated intravitreal injections of antiVEGF agents not only prevent vision loss in most patients but also lead to a significant visual gain in a large number of treated patients. ${ }^{9}, 10$

\section{Steps and preparations in anti-VEGF agent therapy and their association to dry eye}

It is a major concern during ocular surgical treatments that a number of patients often experience postoperative discomfort and grittiness of the eyes and the induction of dry eye syndrome. This is especially common for patients undergoing prolonged chronic treatment that disrupts the ocular surface, such as intravitreal anti-VEGF injections. A recent study performed on 12 patients receiving intravitreal injections of anti-VEGF agents demonstrated that repeated exposure to this ocular treatment can significantly impact the health of the ocular surface of patients leading to gritty eyes and patient discomfort, ${ }^{11}$ prompting the necessity to investigate this link further in detail, in order to identify the potential source(s) of the symptoms.

The main risk in chronic anti-VEGF therapy is the possibility of ocular infection during treatments. Endophthalmitis is a rare but serious complication of intraocular surgery, and the avoidance of this and other forms of ocular infection has been the primary motivation for use of antibacterial and disinfecting agents. In order to prevent serious postoperative effects in patients, endophthalmitis and other forms of ocular infection need to be removed before ocular injection.

\section{Antiseptic agents, their effects on ocular health, and association to dry eye}

As repeated exposure to antiseptic agents occurs during ocular procedures such as anti-VEGF therapy that requires patients to receive monthly injections for up to 2 years, these agents are a likely factor in the development of dry eye syndrome. Therefore, increased attention should be given to the reactions these agents cause during or after their use on ocular surfaces.

Some ophthalmologists use pre- or postoperative topical antibiotics. ${ }^{12}$ The drug itself, or other components of the drug solution, could potentially induce toxic side effects to the ocular surface cells. As discussed in more detail by 
Mantelli et al, ${ }^{13}$ the detrimental role of topical therapies on the ocular surface and the thin border between their therapeutic and toxic effects may arise from both the preservatives and other components in the preparations depending on dose, frequency, and duration of treatment. These negative effects appear to be more easily elicited in predisposed patients, such as hyperreactive patients with underlying ocular allergy, elderly patients with dry eye or glaucoma, and patients affected by severe ocular diseases in need of chronic therapy. The study ${ }^{13}$ concluded that although signs and symptoms of ocular surface damage are variable and often shared by all these conditions, recent in vitro, in vivo, and clinical studies suggest that ocular surface reactions triggered by eye drop treatments are primarily mediated by three different specific pathogenetic mechanisms: 1) allergic reactions induced by hypersensitivity to preservatives and/or medications; 2 ) dry eye-like reactions induced by alteration of the tear film by preservatives; and 3) direct epithelial damage induced by a chronic and/or cumulative use of topical treatments.

Another study addressing the cytotoxicity of six topical antibiotic eyedrops, including Bestron ${ }^{\circledR}$ (cefmenoxime) and Cravit $^{\circledR}$ (levofloxacin), on different cultured ocular surface cell lines demonstrated that when diluted 10 times, cell viability was $>80 \% .{ }^{14}$ However, when diluted only twofold before application to cells, the viability of cells decreased to $43 \%$. Even though cell culture data cannot be directly extrapolated to in vivo findings due to rapid dilution of solution by preexisting tears, as well as clearance by the tear drainage, repeated exposure to these chemicals may sensitize the ocular surface cells and over time cause unwanted side effects.

Moreover, as most antibiotic solutions include preservatives, one should be aware of the damaging effects and concomitant ocular health issues associated. Indeed, Mencucci et $\mathrm{a}^{15}$ compared the cytotoxic effects of preserved versus unpreserved commercially available ophthalmic preparations of fluoroquinolones on human keratocytes in vitro, and their results confirmed the cytotoxic role of preservatives. Singleuse preservative-free preparations of ocular antibiotics may help reduce the discomfort and pain associated with repeated topical antibiotic eye drops use in the longer term.

The use of iodine as an antiseptic agent during ocular surgery is another probable cause of grittiness and dry eye syndrome after repeated ocular treatments. Iodinecontaining solutions have been demonstrated to be the most effective perioperative prophylaxis against the occurrence of endophthalmitis or other postoperative complications. Iodine is the only agent that is consistently active against gram-positive and gram-negative bacteria, spores, amoebic cysts, fungi, protozoa, yeast, drug-resistant bacteria, and viruses. ${ }^{16}$ Ocular irritation caused by povidone-iodine occurs when solutions with high levels of free molecular iodine and moderate to high levels of total iodine are used.

Conflicting experimental and clinical data exist on the abrasive and toxic effects of the use of topical iodine in the ocular surface, especially in cases of repeated exposure. This arises mainly from the use of differing concentrations, incubation times, number of exposure times, experimental systems, etc., between the studies, making it difficult to draw specific conclusions and provide effective guidelines to doctors performing the surgery. For instance, although povidone-iodine has been recognized as a safe, effective broad-spectrum, biocidal agent for many years, ${ }^{17,18}$ there are a handful of studies demonstrating the abrasiveness of iodine treatment leading to corneal epithelial and endothelial cell damage after treatment with povidone-iodine with concentrations higher than $1 \%-5 \%$ and treatment longer than 2 minutes. ${ }^{19-21}$

Another study by Shibata et al ${ }^{22}$ highlighted the cytotoxicity of povidone-iodine solution to human corneal epithelial cells, linking its cytotoxicity to a number of factors, such as the low $\mathrm{pH}$ of iodine, available iodine concentration, osmolarity, and exposure to lauromacrogol, a surfactant used in povidone-iodine. Alternatively, they investigated the possible negative side effects of another antiseptic, polyvinyl alcoholiodine solution (PAI), and their results revealed that PAI exhibited a much lower cytotoxicity than povidone-iodine and displayed a similar effect as saline on cell viability.

The administration guideline for povidone-iodine 5\% Sterile Ophthalmic Prep Solution(Betadine; Alcon, Fortsworth, TX, USA) describes saturation of the lids, brow, and cheek in ever-widening circles until the entire surgical field is covered. The cornea, conjunctiva, and palpebral fornices are then to be irrigated and left in contact with the solution for 2 minutes prior to flushing with a sterile saline solution. As none of the studies discussed earlier precisely replicated this application technique, conclusions about the in vivo efficacy of higher dilutions of povidone-iodine remain uncertain. ${ }^{16}$ Furthermore, one has to consider that the majority of patients receiving the repeated exposure to povidone-iodine during, eg, anti-VEGF therapy, are possibly preconditioned to a sensitized state of the ocular surface, making the effects of iodine and the irritation and dry eye caused by its use more pronounced. Altogether, these findings justify further investigation of the safety and efficacy of povidone-iodine for topical prophylaxis prior to intravitreal anti-VEGF therapy. 
As discussed earlier, a number of different factors, such as the antibiotics, preservatives in those antibiotics, povidoneiodine, and anti-VEGF agents, may significantly contribute to the sensitization of the ocular surface and induce the formation of dry eye syndrome. The role that each of these factors plays in producing the experienced toxic side effects and the extent of each factor have yet to be clinically determined. It is clear, however, that repeated exposure to any possible irritants can affect and influence the outcome of ophthalmic treatments. Based on the earlier analysis, further investigation on specifically the repeated topical use of iodine during these treatments should be examined.

\section{The need to consolidate and track dry eye reports}

It would be immensely helpful to practicing ophthalmologists to track reports of dry eye in order to systematically address the issue of dry eye syndrome after repeated ocular treatments to identify the true scope of the condition. Nevertheless, as different frequencies of injections/treatments, different procedures, and antiseptic methods are practiced between different doctors and clinics, it could present a huge challenge to consolidate available data into a comprehensive systematic and objective analysis. It could also prove useful to evaluate and document the patient's preexisting tear film and ocular surface status before the ocular surgery or ocular injections, as better awareness and pre-procedural optimization might play a role in improved management of symptoms afterward.

For example, the application of the Ocular Surface Disease Index (OSDI) questionnaire in combination with a full examination of the patient's ocular health before surgery could be one possible alternative to address this. For instance, a study by Werdich et $\mathrm{al}^{23}$ identified that within the 50 patients included in the study, $32 \%$ had a history of dry eye or blepharitis prior to receiving intravitreal injections. Dry eye symptoms and signs are currently evaluated by an OSDI questionnaire that includes ocular discomfort, visual function, and environmental triggers. ${ }^{24}$ OSDI questionnaire is a trusted test ophthalmologists have been using for years to help assess chronic dry eye (CDE) disease. However, as discomfort and pain are a subjective phenomenon that varies from patient to patient, it may provide somewhat misleading non-objective results, making it hard to analyze patient data in cohorts.

Due to some lack of objectivity in the OSDI test, it is advisable to pair it with other clinical assessments and anatomical testing, including fluorescent staining, conjunctival vascularization evaluation, meibomian gland function evaluation, visual acuity, and intraocular pressure to acquire a thorough objective understanding of the state of the patient's ocular health.

\section{Treatment strategies for dry eye}

Currently, the relief of dry eye syndrome lies in the prescription of lubricative eye drops that alleviate the symptoms and discomfort post treatment. Different patients may have different needs for the characteristics of the eye drops, such as reaction to tear acidity, viscosity, and lipid- or waterbased. Patients often have to work out by trial and error to find the optimal eye drop that gives maximal symptomatic relief.

To address the postoperative treatment of dry eye, we should be looking at the overall causes and effects of dry eye pathology. A paper by Baudouin et $\mathrm{l}^{25}$ elegantly describes the vicious cycle of dry eye pathology and highlights the key factors playing into the development and progression of the syndrome, pinpointing tear film instability and tear hyperosmolarity as the principal steps indicative of an onset of the disease. To prevent the progression of the disease, one should thus focus on measuring and treating the symptoms of tear hyperosmolarity, as the first line of defense against dry eye syndrome.

As hyperosmolarity is a key event in the pathology of dry eye, it should be used as a marker for testing, diagnosis, and follow-up for chronic ocular treatments to identify the presence of the dry eye disease. Several studies have shown that osmolarity correlates better than any other clinical test with the dry eye disease severity. ${ }^{26,27}$ There are different methods available for measuring the tear osmolarity, including freezing point depression, vapor pressure, and electrical impedance. For example, a portable in situ osmometer (TearLab ${ }^{\mathrm{TM}}$, TearLab Corporation, San Diego, CA, USA) measures electrical impedance of nanoliter volumes of tear fluid directly from the eye. As technology evolves, more of these types of on-site analysis tools could become available in the market that may prove to be useful for diagnosing dry eye.

Once symptoms of dry eye have developed, physicians should also focus on the management of tear hyperosmolarity to break the vicious cycle of dry eye syndrome. One strategy could be the inclusion of osmoprotectant compatible solutes, such as erythritol, glycerol, trehalose, taurine, and L-carnitine, inside the artificial tear substitutes. These osmoprotectants stabilize a tear protein's peptide backbone, encourage its correct folding and functioning despite potentially damaging changes in temperature and pressure, and thus reduce 
the propagation of external stresses on to the cell and its molecular machinery. ${ }^{28,29}$

In a large uncontrolled observational study $(n=5,277)$, usage of osmoprotectant-inclusive eye drops was associated with improvements in symptoms and signs of dry eye syndrome compared with baseline. ${ }^{30}$ However, it is important to recognize that the osmoprotective effect depends on how much osmoprotectant actually reaches the cells and how long it is retained there. Small molecules, such as glycerol and erythritol, can readily enter the cells but are also quick to leave. On the contrary, larger molecules, such as L-carnitine, will enter the cells more slowly but are also retained for longer periods of time. Knowing this, future design in effective osmoprotectant-including lubricative drops should perhaps combine the beneficial effects of both compounds in one formulation to increase the overall protective effect against dry eye.

Furthermore, considering that dry eye is a multifactorial disease of the tears and ocular surface based on the definition by the 2007 International Dry Eye Workshop (DEWS), aside from hyperosmolarity of the tear film, inflammation of the ocular surface is a contributing factor as well. ${ }^{31}$ According to findings by Hessen and Akpek, ${ }^{32}$ identification of inflammation as a major factor in dry eye helped make a tremendous step forward in the description and treatment of the condition. Currently, a number of anti-inflammatory treatments are being used for the management of dry eye, including cyclosphorin A, tacrolimus, corticosteroids, tetracycline derivatives, autologous serum, and interleukin-1 receptor antagonist (IL-1Ra). These agents inhibit the expression of inflammatory mediators on the ocular surface, hence restoring the secretion of a healthy tear film and reducing the signs and symptoms. Other novel anti-inflammatory medications are still in development or in clinical trial phases.

In addition, hyperosmolarity and the concurrent expression of pro-inflammatory cytokines may lead to drastic morphological changes in ocular cells. Hyperosmolarity has been shown to be responsible for reduced goblet cell density in the interpalpebral bulbar conjunctiva, ${ }^{33}$ which in return may be responsible for an unstable tear film. However, tear film instability can also happen even without the occurrence of hyperosmolarity but through other etiologies such as xerophthalmia, ocular allergy, topical preservative use, and contact lens wear. Preservatives in ophthalmic solutions, eg, have been demonstrated to reduce the viability and barrier function of human corneal epithelial cells. ${ }^{34}$ Moreover, since AMD is an age-related disease, one has to emphasize that the prolonged discomfort and elevated occurrence of post-surgical complications may also arise from the lower healing potential of the ocular epithelium in elderly and preconditioned (eg, diabetic) patients. One possibility for the deficiency in healing could arise from the decreased number of limbal stem cells present in the eye in those patients, resulting in abnormalities in the corneal surface, "conjunctivalization" of the cornea with vascularization, appearance of goblet cells, and an irregular and unstable epithelium, which leads to ocular discomfort and reduced vision. ${ }^{35}$

As dry eye syndrome is such a multifaceted disease, it is useful to address the various aspects of dry eye syndrome when prescribing treatment to patients. Overall, it would be very helpful for the industry to work out which type of treatment formulation is most suited for a particular type and severity of dry eye syndrome.

\section{Conclusion}

As age and age-related diseases play strongly into the development of dry eye syndrome, it is difficult to identify the extent of the effects that intravitreal injections or adjunct ocular treatments play into the development of dry eye syndrome after the patients have received their therapies. Concerns that elderly populations experience multiple other diseases, such as diabetes, hypertension, hormonal changes, and conditions such as damaged nerve ends that feed into the predisposition of symptoms that cause dry eye syndrome, make it difficult to accurately assess the role that each therapy or treatment procedure plays on inducing dry eye.

However, it is highly probable that preexisting conditions make patients more vulnerable to developing dry eye during treatment. Thus, it would improve patient outcomes if more caution is taken into consideration in terms of the exact procedures and agents used during the treatments to prevent or reduce the development of dry eye. As discussed earlier, there is a need for a prospective clinical study to address the issues raised in this paper that may help develop standardized effective guidelines for maximizing the outcome of the treatment while minimizing or balancing the possible negative side effects (ie, development of endophthalmitis, dry eye syndrome, or other postoperative complications).

In summary:

1) We suggest that closer attention be paid to the precondition of the patients' overall health and the ocular health status before any surgical intervention. We propose that doctors evaluate this using a combination of the OSDI test and eye examination to identify the current condition, predisposition to dry eye, normal eyelid microflora, 
possible resistance to prolonged use of postoperative antibiotics, and other possible risks that may affect the outcome of the treatment.

2) We can conclude that current research does not recommend eliminating the iodine treatment as an antiseptic agent before surgery. However, we have here highlighted the concern of the use of 5\% and higher concentration of povidone-iodine and would endorse a more thorough clinical study into the effects and efficacy of lower dose $(<5 \%)$ and/or reduced treatment time ( $<2$ minutes) of povidone-iodine treatment to reduce the side effects of postoperative dry eye.

3) If preoperative antibiotics are used, shorter course using preservative-free preparations is preferred. However, antibiotic resistance should also be considered when using topical antibiotics.

4) Patient feedback and follow-up examinations are crucial in capturing data on efficacy of treatments and further evaluation of proposed standard for procedures.

Through this review, we hope to urge both clinicians and industry to look into the topics highlighted here, specifically the direct or indirect association to dry eye syndrome. With concerted efforts, we can formulate a competent standard of procedure to fulfill the needs of the patients and combat the post treatment ocular side effects.

To help us in conducting this review, we have searched PubMed for published associations of dry eye to intravitreal injection, using the following search terms: dry eye syndrome, intravitreal injection, anti-VEGF agents, ocular health, and ocular surface.

\section{Acknowledgment}

The authors thank Dr Stephen Pflugfelder of the Department of Ophthalmology, Baylor College of Medicine, Houston, TX, USA, for his valuable input in this manuscript.

\section{Disclosure}

The authors report no conflicts of interest in this work.

\section{References}

1. Miljanovic B, Dana R, Sullivan DA, Schaumberg DA. Impact of dry eye syndrome on vision-related quality of life. Am J Ophthalmol. 2007; 143(3):409-415.

2. Calonge M, Enriquez-de-Salamanca A, Diebold Y, et al. Dry eye disease as an inflammatory disorder. Ocul Immunol Inflamm. 2010;18(4): 244-253.

3. Yeh S, Song XJ, Farley W, Li DQ, Stern ME, Pflugfelder SC. Apoptosis of ocular surface cells in experimentally induced dry eye. Invest Ophthalmol Vis Sci. 2003;44(1):124-129.

4. Congdon NG, Friedman DS, Lietman T. Important causes of visual impairment in the world today. JAMA. 2003;290(15):2057-2060.
5. Mukamal R [webpage on the Internet]. Avastin, Eylea and Lucentis What's the Difference? 2015. Available from: http://www.aao.org/ eye-health/diseases/avastin-eylea-lucentis-difference. Accessed November 14, 2016.

6. Yancopoulos GD. Clinical application of therapies targeting VEGF. Cell. 2010;143(1):13-16.

7. Ferrara N, Hillan KJ, Gerber HP, Novotny W. Discovery and development of bevacizumab, an anti-VEGF antibody for treating cancer. Nat Rev Drug Discov. 2004;3(5):391-400.

8. Ferrara N, Damico L, Shams N, Lowman H, Kim R. Development of ranibizumab, an anti-vascular endothelial growth factor antigen binding fragment, as therapy for neovascular age-related macular degeneration. Retina. 2006;26(8):859-870.

9. Rosenfeld PJ, Brown DM, Heier JS, et al; MARINA Study Group. Ranibizumab for neovascular age-related macular degeneration. N Engl J Med. 2006;355(14):1419-1431.

10. Brown DM, Kaiser PK, Michels M, et al; ANCHOR Study Group. Ranibizumab versus verteporfin for neovascular age-related macular degeneration. $N$ Engl J Med. 2006;355(14):1432-1444.

11. Srinagesh V, Ellenberg D, Scharper PH, Etter J. Intravitreal Dry Eye Study. Invest Ophthalmol Vis Sci. 2014;55(13):3696-3696.

12. Garg P, Roy A, Sharma S. Endophthalmitis after cataract surgery: epidemiology, risk factors, and evidence on protection. Curr Opin Ophthalmol. 2017;28(1):67-72.

13. Mantelli F, Tranchina L, Lambiase A, Bonini S. Ocular surface damage by ophthalmic compounds. Curr Opin Allergy Clin Immunol. 2011;11(5): 464-470.

14. Ayaki M, Iwasawa A, Niwano Y. In vitro assessment of the cytotoxicity of six topical antibiotics to four cultured ocular surface cell lines. Biocontrol Sci. 2012;17(2):93-99.

15. Mencucci R, Paladini I, Pellegrini-Giampietro DE, Menchini U, Scartabelli T. In vitro comparison of the cytotoxic effects of clinically available ophthalmic solutions of fluoroquinolones on human keratocytes. Can J Ophthalmol. 2011;46(6):513-520.

16. Abelson MB [webpage on the Internet]. Iodine: An Elemental Force against Infection; 2009. Available from: https://www.reviewofophthalmology.com/article/iodine-an-elemental-force-against-infection. Accessed July 3, 2017.

17. Speaker MG, Menikoff JA. Prophylaxis of endophthalmitis with topical povidone-iodine. Ophthalmology. 1991;98(12):1769-1775.

18. Mino de Kaspar H, Chang RT, Singh K, Egbert PR, Blumenkranz MS, Ta CN. Prospective randomized comparison of 2 different methods of $5 \%$ povidone-iodine applications for anterior segment intraocular surgery. Arch Ophthalmol. 2005;123(2):161-165.

19. Jiang J, Wu M, Shen T. The toxic effect of different concentrations of povidone iodine on the rabbit's cornea. Cutan Ocul Toxicol. 2009;28(3): 119-124.

20. Naor J, Savion N, Blumenthal M, Assia EI. Corneal endothelial cytotoxicity of diluted povidone - iodine. J Cataract Refract Surg. 2001;27(6): 941-947.

21. Pels E, Vrensen GF. Microbial decontamination of human donor eyes with povidone-iodine: penetration, toxicity, and effectiveness. $\mathrm{Br} J$ Ophthalmol. 1999;83(9):1019-1026.

22. Shibata Y, Tanaka Y, Tomita T, et al. Evaluation of corneal damage caused by iodine preparations using human corneal epithelial cells. Jpn J Ophthalmol. 2014;58(6):522-527.

23. Werdich X, Ruez T, Singh R. Prevalence and severity of blepharitis symptoms and signs amongst patients with age-related macular degeneration. Invest Ophthalmol Vis Sci. 2010;51(13):5122-5122.

24. Li XM, Zhao X, Hu LZ, Wang W. [Clinical observation of dry eye in patients before and after cataract surgery]. Zhonghua yan ke za zhi [Chin J Ophthalmol]. 2007;43(1):10-13. Chinese.

25. Baudouin C, Aragona P, Messmer EM, et al. Role of hyperosmolarity in the pathogenesis and management of dry eye disease: proceedings of the OCEAN group meeting. Ocul Surf. 2013;11(4):246-258.

26. Versura P, Profazio V, Campos EC. Performance of tear osmolarity compared to previous diagnostic tests for dry eye diseases. Curr Eye Res. 2010;35(7):553-564. 
27. Jacobi C, Jacobi A, Kruse FE, Cursiefen C. Tear film osmolarity measurements in dry eye disease using electrical impedance technology. Cornea. 2011;30(12):1289-1292.

28. Alfieri RR, Cavazzoni A, Petronini PG, et al. Compatible osmolytes modulate the response of porcine endothelial cells to hypertonicity and protect them from apoptosis. J Physiol. 2002;540(pt 2):499-508.

29. Cammarata PR, Schafer G, Chen SW, Guo Z, Reeves RE. Osmoregulatory alterations in taurine uptake by cultured human and bovine lens epithelial cells. Invest Ophthalmol Vis Sci. 2002;43(2):425-433.

30. Kaercher T, Buchholz P, Kimmich F. Treatment of patients with keratoconjunctivitis sicca with Optive ${ }^{\mathrm{TM}}$ : results of a multicenter, open-label observational study in Germany. Clin Ophthalmol. 2009;3:33-39.
31. The definition and classification of dry eye disease: report of the Definition and Classification Subcommittee of the International Dry Eye WorkShop (2007). Ocul Surf. 2007;5(2):75-92.

32. Hessen M, Akpek EK. Dry eye: an inflammatory ocular disease. J Ophthalmic Vis Res. 2014;9(2):240-250.

33. Nelson JD, Wright JC. Conjunctival goblet cell densities in ocular surface disease. Arch Ophthalmol. 1984;102(7):1049-1051.

34. Xu M, Sivak JG, McCanna DJ. Comparison of the effects of ophthalmic solutions on human corneal epithelial cells using fluorescent dyes. J Ocul Pharmacol Ther. 2013;29(9):794-802.

35. Dua HS, Azuara-Blanco A. Limbal stem cells of the corneal epithelium. Surv Ophthalmol. 2000;44(5):415-425.
Clinical Ophthalmology

\section{Publish your work in this journal}

Clinical Ophthalmology is an international, peer-reviewed journal covering all subspecialties within ophthalmology. Key topics include: Optometry; Visual science; Pharmacology and drug therapy in eye diseases; Basic Sciences; Primary and Secondary eye care; Patien Safety and Quality of Care Improvements. This journal is indexed on

Submit your manuscript here: http://www.dovepress.com/clinical-ophthalmology-journal

\section{Dovepress}

PubMed Central and CAS, and is the official journal of The Society of Clinical Ophthalmology (SCO). The manuscript management system is completely online and includes a very quick and fair peer-review system, which is all easy to use. Visit http://www.dovepress.com/ testimonials.php to read real quotes from published authors. 